\title{
Bures Distance Function and a Generalization of Sakai's Non-commutative Radon-Nikodym Theorem
}

By

\author{
Huzihiro Araki*
}

\begin{abstract}
For normal positive linear functionals $\mu$ and $\nu$ of a $W^{*}$ algebra $\Re$, the following extension of a noncommutative Radon-Nikodym theorem by Sakai is given.

There exist decompositions $\mu=\mu_{1}+\mu_{2}, \nu=\nu_{1}+\nu_{2}$ such that $\nu_{2}$ is the smallest normal positive linear functional on $\Re$ satisfying $\nu \geqq \nu_{2}$ and $s\left(\nu_{2}\right) \perp s(\mu)$, where $s(\alpha)$ denotes the support projection of $\alpha$, and $\mu_{2}$ is the smallest normal positive linear functional on $\Re$ satisfying $\mu \geqq \mu_{2}$ and $s\left(\mu_{2}\right) \perp s(\nu)$. Further, there exists a non-negative self-adjoint operator $A_{1}=A_{1}(\nu / \mu)$ (in general unbounded) such that $A_{1}=\int \lambda d E_{\lambda}^{1}$ with its spectral projections $E_{\lambda}^{1}$ in $\Re, \lim _{\lambda \downarrow 0} E_{\lambda}^{1}=1-s_{\mu}^{\nu}$ and

$$
\nu\left(s\left(\mu_{1}\right) Q s\left(\mu_{1}\right)\right)=\mu_{1}\left(A_{1} Q A_{1}\right) \equiv \lim _{\lambda, \lambda^{\prime}} \mu_{1}\left(A_{1} E_{\lambda}^{1} Q A_{1} E_{\lambda^{\prime}}^{1}\right)
$$
\end{abstract}

for all $Q \in \Re$, where $s_{\mu}^{\nu}=s\left(\mu_{1}\right)-s\left(\mu_{1}\right) \wedge(1-s(\nu))$. There also exists another non-negative self-adjoint operator $A_{2}=A_{2}(\nu / \mu)$ such that its spectral projections $E_{\lambda}^{2}$ are in $\Re, \lim _{\lambda \downarrow 0} E_{\lambda}^{2}=1-s_{\nu}^{\mu}$ and, for all $Q \in \Re$,

$$
\nu_{1}\left(s_{\nu}^{\mu} Q s_{\nu}^{\mu}\right)=\mu\left(A_{2} Q A_{2}\right) .
$$

They are related by $A_{1}(\nu / \mu) A_{2}(\mu / \nu)=A_{2}(\mu / \nu) A_{1}(\nu / \mu)=s_{\mu}^{\nu}$.

The Bures distance function $d(\mu, \nu)$ is given by

Received June 12, 1972.

* On leave from Research Institute for Mathematical Sciences, Kyoto Univ., Kyoto, Japan. 


$$
\begin{aligned}
d(\mu, \nu)^{2} & =\mu(1)+\nu(1)-2 \mu_{1}\left(A_{1}\right) \\
& =\mu(1)+\nu(1)-2 \mu\left(A_{2}\right) .
\end{aligned}
$$

In any representation $\pi$ of $\Re$, if two vectors $\Psi$ and $\Phi$ satisfy $\omega_{\Psi}=\mu$, $\omega_{\Phi}=\nu$ and $\|\Psi-\Phi\|=d(\mu, \nu)$, where $\omega_{\Psi}$ denotes the vector state by $\Psi$, then there is a decomposition $\pi=\pi_{1} \oplus \pi^{\prime}, \Psi=x_{1} \oplus x^{\prime}, \Phi=y_{1} \oplus y^{\prime}, \omega_{x^{\prime}}=$ $\mu_{2}, \omega_{y^{\prime}}=\nu_{2}, x_{1}$ and $y_{1}$ are cyclic vectors of $\pi_{1}, \pi_{1}\left(s\left(\mu_{1}\right)\right) y_{1}=\pi_{1}\left(A_{1}\right) x_{1}$, $\pi_{1}\left(s_{\nu}^{\mu}\right) y_{1}=\pi_{1}\left(A_{2}\right) x_{1}$, and such that triplet $\pi_{1}, x_{1}$ and $y_{1}$ are unique up to unitary equivalence for given $\mu$ and $\nu$.

\section{$\S 1$. Introduction}

For two normal positive linear functionals $\mu$ and $\nu$ of a $W^{*}$-algebra $\Re$ satisfying $\mu \geqq \nu$, Sakai $[5]$ has shown the existence of a unique $t_{0} \in \Re$ such that $0 \leqq t_{0} \leqq 1$ and

$$
\nu(Q)=\mu\left(t_{0} Q t_{0}\right)
$$

for all $Q \in \Re$. We shall generalize this Radon-Nikodym theorem of Sakai to the case where $\mu \geqq \nu$ does not necessarily hold.

Our investigation originally started from a search for a standard form of vectors $\Psi$ and $\Phi$ such that their vector "states" are $\mu$ and $\nu$ and $\|\Psi-\bar{\Phi}\|$ is minimal. The minimal value of $\|\Psi-\Phi\|$ is defined to be $d(\mu, \nu)$ by Bures [2]. It is easily shown that, if $\mu \geqq \nu$ holds, then $\Phi$ is uniquely given by

$$
\Phi=\pi\left(t_{0}\right) \Psi
$$

and hence

$$
d(\mu, \nu)^{2}=\mu(1)+\nu(1)-2 \mu\left(t_{0}\right) .
$$

We shall first show the existence of a pair $\Psi$ and $\Phi$ giving the mimimal distance for general $\mu$ and $\nu$. An analysis of their mutual relation leads to a generalization of $t_{0}$. The result reduces to the RadonNikodym theorem by Sakai if $\mu \geqq \nu$. 
Notations and Conventions: All representations of $\mathfrak{R}$ in the present work will be normal representations. We denote the set of all normal positive linear functionals on $\Re$ by $S(\Re)$. The expectation functional on $\Re$ by a vector $x$ in a representation space $\mathfrak{L}$ of $\Re$ is denoted by $\omega_{x}$. The support $s(\mu)$ of $\mu \in S(\Re)$ is the smallest projection operator $E \in \Re$ satisfying $\mu(E)=\mu(1)$. The support $s(x)$ of a vector $x$ in $\mathfrak{S}$ relative to a representation $\pi$ of $\Re$ on $\mathfrak{S}$ is the smallest projection $E \in \pi(\Re)$ satisfying $E x=x . \quad E \mathfrak{S}$ is the closure of $\pi(\Re)^{\prime} x$ and $s(x)=\pi\left(s\left(\omega_{x}\right)\right)$. The support $s(\pi)$ of a representation $\pi$ is the smallest central projection $E \in \Re$ satisfying $\pi(E)=1$. $\pi$ is faithful on $\Re s(\pi)$. The support $s(Q)$ of an operator $Q$ is the smallest projection $E$ such that $E Q=Q E=Q . \quad s(Q)=$ $s\left(Q^{*} Q\right) \vee s\left(Q Q^{*}\right)$ and it belongs to $\Re$ if $Q \in \Re$.

Our main results are following theorems:

Theorem 1. Let $\mu, \nu \in S(\Re)$.

(1) There exists a unique decomposition $\mu=\mu_{1}+\mu_{2}, \nu=\nu_{1}+\nu_{2}$ such that $\nu_{2}$ is the largest $\rho \in S(\Re)$ satisfying $\nu \geqq \rho$ and $s(\mu) \perp s(\rho)$, and $\mu_{2}$ is the largest $\rho \in S(\Re)$ satisfying $\mu \geqq \rho$ and $s(\nu) \perp s(\rho)$.

(2) There exists a non-negative self-adjoint operator

$$
A_{1}=A_{1}(\nu / \mu)=\int \lambda d E_{\lambda}^{1}
$$

such that $E_{\lambda}^{1} \in \Re, \lim _{\lambda \downarrow 0} E_{\lambda}^{1}=1-s_{\mu}^{\nu}$ and

$$
\begin{aligned}
\nu\left(s\left(\mu_{1}\right) Q s\left(\mu_{1}\right)\right) & =\nu\left(s_{\mu}^{\nu} Q s_{\mu}^{\nu}\right) \\
& =\mu_{1}\left(A_{1} Q A_{1}\right) \equiv \lim _{\lambda, \lambda^{\prime} \rightarrow+\infty} \mu_{1}\left(A_{1} E_{\lambda}^{1} Q A_{1} E_{\lambda^{\prime}}^{1}\right)
\end{aligned}
$$

where

$$
s_{\mu}^{\nu}=s\left(\mu_{1}\right)-s\left(\mu_{1}\right) \wedge(1-s(\nu))
$$

(3) There exists a non-negative self-adjoint operator

$$
A_{2}=A_{2}(\nu / \mu) \equiv \int \lambda d E_{\lambda}^{2}
$$

such that $E_{\lambda}^{2} \in \Re, \lim _{\lambda \downarrow 0} E_{\lambda}^{2}=1-s_{\nu}^{\mu}, A_{1}(\mu / \nu) A_{2}(\nu / \mu)=s_{\nu}^{\mu}$, and 


$$
\nu_{1}\left(s_{\nu}^{\mu} Q s_{\nu}^{\mu}\right)=\mu\left(A_{2} Q A_{2}\right) .
$$

(4) In a representation $\pi_{1}$ of $\Re$ with a cyclic vector $x_{1}$ satisfying $\omega_{x_{1}}=\mu_{1}$, there exists a unique vector $y_{1}$ such that $\omega_{y_{1}}=\nu_{1}$ and

$$
s\left(x_{1}\right) y_{1}=\pi_{1}\left(A_{1}\right) x_{1} \equiv \lim _{\lambda \rightarrow+\infty} \pi_{1}\left(A_{1} E_{\lambda}^{1}\right) x_{1}
$$

It satisfies

$$
\left\{s\left(y_{1}\right)-s\left(y_{1}\right) \wedge\left(1-s\left(x_{1}\right)\right)\right\} y_{1}=\pi_{1}\left(A_{2}\right) x_{1}
$$

Theorem 2. For any $\mu, \nu \in S(\Re)$,

$$
\begin{aligned}
d(\mu, \nu)^{2} & =\mu(1)+\nu(1)-2 \mu_{1}\left(A_{1}\right) \\
& =\mu(1)+\nu(1)-2 \mu\left(A_{2}\right)
\end{aligned}
$$

where $A_{1}$ and $A_{2}$ are as in Theorem 1 and $\mu_{1}\left(A_{1}\right)=\lim _{\lambda \rightarrow+\infty} \mu_{1}\left(A_{1} E_{\lambda}^{1}\right)$.

For any vectors $\Psi$ and $\Phi$ in a representation $\pi$ of $\Re$ satisfying $\omega_{\Psi}=\mu$, $\omega_{\oplus}=\nu$, and $d(\mu, \nu)=\|\Psi-\Phi\|$, there exists a decomposition $\pi=\pi_{1} \oplus \pi^{1}$, $\Psi=x_{1} \oplus x^{1}, \Phi=y_{1} \oplus y^{1}$, such that $\omega_{x^{1}}=\mu_{2}, \omega_{y^{1}}=\nu_{2}, x_{1}$ and $y_{1}$ are cyclic for $\pi_{1}(\Re)$, the triplet $\pi_{1}, x_{1}$ and $y_{1}$ are unitarily equivalent to $\pi_{1}, x_{1}$ and $y_{1}$ of Theorem 1 (4) and is unique up to unitary equivalence.

Takesaki $([8] \S 15)$ considers the case $s(\mu)=1$. His $h_{0}$ has the same matrix element as our $A_{2}$ on the dense domain $\pi_{\mu}(\Re)^{\prime} \Psi$.

\section{$\S 2 . \quad$ Bures Distance Function}

The Bures distance for $\mu, \nu \in S(\Re)$ is

$$
d(\mu, \nu)=\inf \left\{\|x-y\| ; \omega_{x}=\mu, \omega_{y}=\nu\right\}
$$

where $x$ and $y$ can be in an arbitrary representation space of $\Re$. The following lemma shows that the infimum is actually reached.

Lemma 1. For $\mu \in S(\Re)$, there exist a representation $\pi_{\mu}$ of $\Re$ on $\mathfrak{S}_{\mu}$ and a vector $\Psi$ in $\mathfrak{S}_{\mu}$ such that $\mu=\omega_{\Psi}$ and for any $\nu \in S(\Re)$ there exists $\Phi \in \mathfrak{S}_{\mu}$ satisfying $\omega_{\varpi}=\nu$ and $\|\Psi-\Phi\|=d(\mu, \nu)$. 
Proof. By Proposition 1.6 of Bures [2], there exists a representation $\pi_{B}$ of $\mathfrak{R}$ on $\mathfrak{S}_{B}$ and a vector $x_{B}$ in $\mathfrak{S}_{B}$ such that $\omega_{x_{B}}=\mu$ and

$$
d(\mu, \nu)=\inf \left\{\left\|x_{B}-y\right\| ; y \in \mathfrak{S}_{B}, \omega_{y}=\nu\right\}
$$

for any $\nu \in S(\Re)$.

Let $y_{n}$ be such that $y_{n} \in \mathfrak{S}_{B}, \omega_{y_{n}}=\nu$ and

$$
\lim _{n}\left\|x_{B}-y_{n}\right\|=d(\mu, \nu) .
$$

By weak sequential compactness, there exists a subsequence $n(k)$ and $y \in \mathfrak{S}_{B}$ such that

$$
\mathrm{w}-\lim _{k} y_{n(k)}=y \text {. }
$$

Then

$$
\nu-\omega_{y}=\lim _{k} \omega_{\left(y_{n(k)}-y\right)} \geqq 0 .
$$

Therefore $\nu-\omega_{y} \in S(\Re)$ and there exists $y^{1} \in \mathfrak{S}_{B}$ satisfying $\nu-\omega_{y}=\omega_{y^{1}}$. We also have

$$
\begin{aligned}
\left\|x_{B}-y\right\|^{2} & =\left\|x_{B}\right\|^{2}+\|y\|^{2}-2 \operatorname{Re} \lim \left(x_{B}, y_{n(k)}\right) \\
& =\lim \left\|x_{B}-y_{n(k)}\right\|^{2}-\lim \left\|y_{n(k)}-y\right\|^{2} \\
& =d(\mu, \nu)^{2}-\omega_{y^{1}}(1) .
\end{aligned}
$$

Hence $\mathfrak{S}_{\mu}=\mathfrak{S}_{B} \oplus \mathfrak{S}_{B}, \pi_{\mu}=\pi_{B} \oplus \pi_{B}, \Psi=x_{B} \oplus 0$ and $\Phi=y \oplus y^{1}$ satisfy all the requirements.

Q.E.D.

The next Lemma is not needed in the proof of the main Theorems and is a special case of Theorem 2. We present it here because it gives a motivation for the proof technique in the following sections.

Lemma 2. Let $\mu \in S(\Re), t_{0} \in \Re, t_{0} \geqq 0$, and $\nu(Q)=\mu\left(t_{0} Q t_{0}\right)$ for all $Q \in \Re$. Then

$$
d(\mu, \nu)^{2}=\mu(1)+\nu(1)-2 \mu\left(t_{0}\right)
$$


Proof. Let $\mathfrak{S}_{\mu}, \pi_{\mu}, \Psi$ and $\Phi$ be as in Lemma 1 . Let $\Phi^{\prime}=\pi_{\mu}\left(t_{0}\right) \Psi$. Then $\omega_{\oplus}^{\prime}=\nu$ and

$$
d(\mu, \nu)^{2} \leqq\left\|\Psi-\Phi^{\prime}\right\|^{2}=\mu(1)+\nu(1)-2 \mu\left(t_{0}\right)
$$

Let $\alpha$ be defined on $\pi_{\mu}(\Re) \Phi^{\prime}$ by

$$
\alpha \pi_{\mu}(Q) \Phi^{\prime}=\pi_{\mu}(Q) \Phi, Q \in \Re
$$

Then $\alpha$ is isometric on $\pi_{\mu}(\Re) \Phi^{\prime}$ :

$$
\left\|\pi_{\mu}(Q) \Phi\right\|^{2}=\nu\left(Q^{*} Q\right)=\left\|\pi_{\mu}(Q) \Phi^{\prime}\right\|^{2} .
$$

Hence $\alpha$ is well-defined on $\pi_{\mu}(\Re) \Phi$, linear there and $\|\alpha\| \leqq 1$. Let $s^{\prime}(\Phi)$ be the projection on the closure of $\pi_{\mu}(\Re) \Phi$. Then $s^{\prime}(\Phi) \in \pi_{\mu}(\Re)^{\prime}$ and $\hat{\alpha} \equiv \alpha s^{\prime}(\Phi) \in \pi_{\mu}(\Re)^{\prime}$. We have

$$
\begin{aligned}
|(\Psi, \Phi)| & =\left|\left(\Psi, \hat{\alpha} \Phi^{\prime}\right)\right| \\
& =\left|\left(\pi_{\mu}\left(t_{0}\right)^{1 / 2} \Psi, \hat{\alpha} \pi_{\mu}\left(t_{0}\right)^{1 / 2} \Psi\right)\right| \\
& \leqq\|\hat{\alpha}||\| \pi_{\mu}\left(t_{0}\right)^{1 / 2} \Psi \|^{2} \leqq \mu\left(t_{0}\right)
\end{aligned}
$$

Hence

$$
\begin{aligned}
d(\mu, \nu)^{2} & =\mu(1)+\nu(1)-2 \operatorname{Re}(\Psi, \Phi) \\
& \geqq \mu(1)+\nu(1)-2 \mu\left(t_{0}\right) .
\end{aligned}
$$

Q.E.D.

Remark. Lemma 2 gives the uniqueness of $t_{0}$ satisfying

(i) $t_{0} \in \Re, t_{0} \geqq 0$,

(ii) $\mu\left(t_{0} Q t_{0}\right)=\nu(Q), Q \in \Re$,

(iii) $s\left(t_{0}\right) \leqq s(\mu)$,

for given $\mu$ and $\nu$ by the following argument.

Consider the representation $\pi_{\mu}$ of $\mathfrak{R}$ on $\mathfrak{C}_{\mu}$ with a cyclic vector $\Omega_{\mu}$ such that $\omega_{\Omega_{\mu}}=\mu$. Assume that $t_{0}$ and $t_{0}^{\prime}$ satisfy (i)-(iii). From the proof of Corollary, which gives the uniqueness of $y$ satisfying $\omega_{y}=\nu$, 
$\omega_{x}=\mu, d(\mu, \nu)=2(1-(x, y)) \quad($ for given $\mu, \nu, x)$, we obtain $\pi_{\mu}\left(t_{0}\right) \Omega_{\mu}$ $=\pi_{\mu}\left(t_{0}^{\prime}\right) \Omega_{\mu}$. Hence $\pi_{\mu}\left(t_{0}\right) Q^{\prime} \Omega_{\mu}=\pi_{\mu}\left(t_{0}^{\prime}\right) Q^{\prime} \Omega_{\mu}$ for any $Q^{\prime} \in \pi_{\mu}(\Re)^{\prime}$. Therefore $\pi_{\mu}\left(t_{0}-t_{0}^{\prime}\right) s\left(\Omega_{\mu}\right)=0$. Since $s\left(\Omega_{\mu}\right)=\pi_{\mu}(s(\mu))$ and the representation $\pi_{\mu}$ is faithful at least for $s(\mu) \Re s(\mu)$, we have $s(\mu)\left(t_{0}-t_{0}^{\prime}\right) s(\mu)=0$. By (iii), we have $t_{0}=t_{0}^{\prime}$.

Corollary. Let $\mu, \nu, t_{0}$ be as in Lemma 2. Let $\pi$ be a representation of $\mathfrak{R}$ on $\mathfrak{S}$ and $\Psi, \Phi \in \mathfrak{S}$ satisfy $\omega_{\Psi}=\mu, \omega_{\mathscr{\Phi}}=\nu$ and $d(\mu, \nu)^{2}=$ $\|\Psi-\Phi\|^{2}$. Then

$$
\Phi=\pi\left(t_{0}\right) \Psi
$$

Proof. From the preceding proof, we have

$$
\begin{aligned}
\operatorname{Re}(\Psi, \Phi) & =\operatorname{Re}\left(\pi\left(t_{0}\right)^{1 / 2} \Psi, \hat{\alpha} \pi\left(t_{0}\right)^{1 / 2} \Psi\right) \\
& =\left\|\pi\left(t_{0}\right)^{1 / 2} \Psi\right\|^{2}
\end{aligned}
$$

Hence $|\hat{\alpha}| \leqq 1$ implies

$$
\hat{\alpha} \pi\left(t_{0}\right)^{1 / 2} \Psi=\pi\left(t_{0}\right)^{1 / 2} \Psi .
$$

Since $\hat{\alpha} \in \pi(\Re)^{\prime}$, we have

$$
\bar{\Phi}=\pi\left(t_{0}\right)^{1 / 2} \hat{\alpha} \pi\left(t_{0}\right)^{1 / 2} \Psi=\pi\left(t_{0}\right) \Psi .
$$

Q.E.D.

Remark. If $\Re$ is a type I factor, $\mu(Q)=\operatorname{tr}(\rho Q), \nu(Q)=\operatorname{tr}(\sigma Q)$ for $Q \in \Re, \rho \geqq \sigma, \rho>0$, then

$$
t_{0}=\left(\rho^{-1 / 2}\left|\sigma^{1 / 2} \rho^{1 / 2}\right| \rho^{-1 / 2}\right)^{-}
$$

and

$$
\mu\left(t_{0}\right)=\operatorname{tr}\left|\sigma^{1 / 2} \rho^{1 / 2}\right|
$$

where $|\beta|$ denotes $\left(\beta^{*} \beta\right)^{1 / 2}$. 


\section{§3. Construction of $\boldsymbol{A}$}

The following construction of $A_{0}$ is similar to the method of Takesaki [8]. $\mathfrak{S}_{0}$ is not assumed to be separable.

Lemma 3. Let $x_{0}$ be a cyclic and separating vectors for a von Neuman algebra $\Re_{0}$ on $\mathfrak{S}_{0}$ and $z$ be a separating vector for $\mathfrak{R}_{0}$ satisfying

$$
\left(x_{0}, Q z\right) \geqq 0
$$

for all $Q \geqq 0, Q \in \Re_{0}^{\prime}$. Then there exists a positive self-adjoint operator

$$
A_{0}=\int_{0}^{\infty} \lambda d E_{\lambda}^{0}
$$

such that $E_{\lambda}^{0} \in \Re_{0}, z=A_{0} x_{0}, E_{+0}^{0} \equiv \lim _{\lambda \downarrow 0} E_{\lambda}^{0}=0$.

Proof. Let $S$ be defined on $\mathfrak{D}=\Re_{0}^{\prime} x_{0}$ by

$$
S Q x_{0}=Q z, Q \in \Re_{0}^{\prime}
$$

Since $x_{0}$ is cyclic for $\Re_{0}, Q x_{0}=0$ for $Q \in \Re_{0}^{\prime}$ implies $Q=0$ and hence $Q z=0$. Therefore $S$ is well-defined, linear operator. Since $x_{0}$ is separating for $\Re_{0}$, the domain $\mathfrak{D}=\mathfrak{R}_{0}^{\prime} x_{0}$ of $S$ is dense.

By assumption (3.1), $\left(x_{0},(c-Q) *(c-Q) z\right)$ is real for $Q \in \Re_{0}^{\prime}$ and any complex number $c$. This implies that

$$
\left(x_{0}, Q z\right)=\left(x_{0}, Q^{*} z\right)^{*}=\left(z, Q x_{0}\right)
$$

Therefore for $Q_{1}, Q_{2} \in \Re_{0}^{\prime}$

$$
\begin{aligned}
\left(Q_{2} x_{0}, S Q_{1} x_{0}\right) & =\left(x_{0}, Q_{2}^{*} Q_{1} z\right) \\
& =\left(z, Q_{2}^{*} Q_{1} x_{0}\right) \\
& =\left(S Q_{2} x, Q_{1} x_{0}\right) .
\end{aligned}
$$

Hence $S$ is symmetric. $S$ is non-negative on $\mathfrak{D}$ by (3.1).

$(D)$ is obviously invariant under $\mathfrak{R}_{0}^{\prime}$. For $Q, Q_{1}, Q_{2} \in \Re_{0}^{\prime}$, 


$$
\begin{gathered}
\left(Q_{2} x_{0}, S Q Q_{1} x_{0}\right)=\left(Q_{2} x_{0}, Q Q_{1} z\right) \\
=\left(Q^{*} Q_{2} x_{0}, Q_{1} z\right) \\
=\left(Q^{*} Q_{2} x_{0}, S Q_{1} x_{0}\right) \\
=\left(Q_{2} x_{0}, Q S Q_{1} x_{0}\right) .
\end{gathered}
$$

Hence $S$ commutes with any $Q$ in $\Re_{0}^{\prime}$.

We now consider the Friedrichs extension of $S$.

Let

$$
\left(\Psi_{1}, \Psi_{2}\right)_{\Re}=\left(\Psi_{1}, S \Psi_{2}\right)+\left(\Psi_{1}, \Psi_{2}\right)
$$

for all $\Psi_{1}, \Psi_{2} \in \mathfrak{D}$. Since

$$
\left(Q x_{0}, Q x_{0}\right)_{\Re}=\left(x_{0}, Q^{*} Q z\right)+\left\|Q x_{0}\right\|^{2}>0
$$

for non-zero $Q \in \Re_{0}^{\prime},\left(\Psi_{1}, \Psi_{2}\right)$ is an inner product on $\mathfrak{D}$. Let $\Re$ be its completion, which is a Hilbert space with $\left(\Psi_{1}, \Psi_{2}\right)_{\Re}$ as an inner product. Let $\alpha$ be the mapping from $Q x_{0}$ in $\Re$ to $Q x_{0}$ in $\mathfrak{S}_{0}$. It is densely defined, linear and $|\alpha| \leqq 1$. Let $\bar{\alpha}$ be its closure.

Since $\mid i Q \|-Q \geqq 0$ for any self-adjoint $Q$, we have

$$
\left(Q_{1} x_{0},(\|Q\|-Q) Q_{1} z\right) \geqq 0
$$

for any $Q_{1} \in \Re_{0}^{\prime}$. Replacing $Q$ by $Q^{*} Q$, we obtain

$$
\left\|Q Q_{1} x_{0}\right\|_{\Re}^{2} \leqq\left\|Q^{*} Q\right\|\left\|Q_{1} x_{0}\right\|_{\Omega}^{2}=\|Q\|^{2}\left\|Q_{1} x_{0}\right\|_{\Omega}^{2}
$$

Therefore $\alpha^{-1} Q \alpha$ is linear and bounded on $\alpha^{-1} \mathfrak{D}$. Let $\pi_{\Re}(Q)$ be its closure on $\Re$. $\alpha \pi_{\Re}(Q)=Q \alpha$ on $\mathfrak{D}$ implies

$$
\bar{\alpha} \pi_{\Re}(Q)=Q \bar{\alpha}
$$

$\pi_{\Re}$ is clearly a $*$ representation of $\Re_{0}^{\prime}$. If $Q_{\alpha}$ is a non-decreasing monotonous net in $\Re_{0}^{\prime}$ with $\lim _{\alpha \uparrow} Q_{\alpha}=Q$, then $\lim _{\alpha \uparrow}\left\|\pi_{\Omega}\left(Q-Q_{\alpha}\right) \Psi\right\|_{\Omega}^{2}=0$ for $\Psi \in$ $\alpha^{-1} \mathfrak{D}$ and hence for $\Psi \in \Re$. Therefore $\pi_{\Re}$ is normal.

From the Schwarz inequality 


$$
\left|\left(\Psi_{1}, S \Psi_{2}\right)\right|^{2} \leqq\left(\Psi_{1}, S \Psi_{1}\right)\left(\Psi_{2}, S \Psi_{2}\right)
$$

for $\Psi_{1}, \Psi_{2} \in \mathfrak{D}$ and the majorization

$$
(\alpha \Psi, S \alpha \Psi) \leqq\|\Psi\|_{\Omega}^{2}
$$

we obtain the existence of a bounded non-negative self-adjoint $T$ on $\Omega$ such that $1 \geqq T$ and

$$
\left(\bar{\alpha} \Psi_{1}, S \alpha \Psi_{2}\right)=\left(\Psi_{1}, T \Psi_{2}\right)_{\Re}
$$

for all $\Psi_{2} \in \alpha^{-1} \mathfrak{D}, \Psi_{1} \in \Re$.

Since $S$ commutes with $Q \in \Re_{0}^{\prime}$, we have from (3.5) and (3.6)

$$
\left(\Psi_{1}, T \pi_{\Omega}(Q) \Psi_{2}\right)_{\Re}=\left(\Psi_{1}, \pi_{\Omega}(Q) T \Psi_{2}\right)_{\Re}
$$

for all $\Psi_{1} \in \Re$ and $\Psi_{2} \in \alpha^{-1} \mathfrak{D}$. Hence $T \in \pi_{\Re}\left(\Re_{0}^{\prime}\right)^{\prime}$.

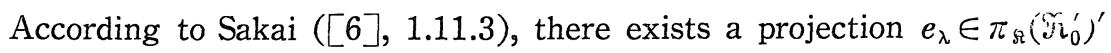
for each real $\lambda$, having the following properties:

(1) $e_{\lambda} \leqq e_{\lambda^{\prime}}$ if $\lambda \leqq \lambda^{\prime}$.

(2) $\lim _{\lambda_{n} \uparrow \lambda} e_{\lambda_{n}}=e_{\lambda}$.

(3) $e_{1+\varepsilon}=1$ for $\varepsilon>0$ and $e_{0}=0$.

(4) $T=\int_{0}^{\infty} \lambda d e_{\lambda}$.

Let the closure of $\bar{\alpha} e_{\lambda} \Re$ be $\mathfrak{S}_{\lambda}$ and the projection onto $\mathfrak{S}_{f(\lambda)}$ be $E_{\lambda}^{0}$ where $f(\lambda)=(1+\lambda)^{-1} \lambda . \quad f$ is a monotonously increasing function on $[0, \infty)$ with the range $[0,1)$. From (1), we have

$$
E_{\lambda}^{0} \leqq E_{\lambda^{\prime}}^{0} \quad \text { if } \lambda \leqq \lambda^{\prime} .
$$

From (2), we have

$$
\lim _{\lambda_{n} \uparrow \lambda} E_{\lambda_{n}}^{0}=E_{\lambda}^{0}
$$

From (3), we have

$$
E_{0}^{0}=0 \text {. }
$$

For $\Psi \in e_{\lambda} \Omega$ and $Q \in \Re_{0}^{\prime}$, we have 


$$
Q \bar{\alpha} \Psi=\bar{\alpha} \pi_{\Re}(Q) \Psi \in \bar{\alpha} e_{\lambda} \Re \subset \oiint_{\lambda}
$$

due to (3.5) and $e_{\lambda} \in \pi_{\Re}\left(\Re_{0}^{\prime}\right)^{\prime}$. Hence $Q \Psi \in \mathscr{S}_{\lambda}$ for any $\Psi \in \mathfrak{C}_{\lambda}, Q \in \Re_{0}^{\prime}$ and hence

$$
E_{\lambda}^{0} \in \Re_{0}
$$

From the definition (3.4), we have for $\Psi_{2} \in \alpha^{-1} \mathfrak{D}$,

$$
\left(\Psi_{1}, \Psi_{2}\right)_{\Omega}=\left(\bar{\alpha} \Psi_{1}, S \alpha \Psi_{2}\right)+\left(\bar{\alpha} \Psi_{1}, \alpha \Psi_{2}\right)
$$

for all $\Psi_{1} \in \alpha^{-1} \mathfrak{D}$ and hence for all $\Psi_{1} \in \Omega$ by continuity. If $\bar{\alpha} \Psi_{1}=0$, then $\left(\Psi_{1}, \Psi_{2}\right)_{\Re}=0$ for all $\Psi_{2}$ in the dense subset $\alpha^{-1} \mathfrak{D}$ of $\Omega$ and hence $\Psi_{1}=0$. Namely the kernel of $\bar{\alpha}$ is 0 .

From (3.11) and (3.6), we have

$$
\begin{aligned}
\left(\bar{\alpha} \Psi_{1}, \bar{\alpha} \Psi_{2}\right) & =\left(\Psi_{1}, \Psi_{2}\right)_{\Re}-\left(\bar{\alpha} \Psi_{1}, S \bar{\alpha} \Psi_{2}\right) \\
& =\left(\Psi_{1},(1-T) \Psi_{2}\right)_{\Re}
\end{aligned}
$$

for $\Psi_{1}, \Psi_{2} \in \alpha^{-1} \mathfrak{D}$ and hence for all $\Psi_{1}, \Psi_{2} \in \Re$ by continuity. From this equality, we obtain the following three conclusions.

(i) If $(1-T) \Psi=0$, then from (3.12) with $\Psi_{1}=\Psi_{2}=\Psi$, we obtain $\Psi=0$. Hence $e_{1}=1$ and

$$
\lim _{\lambda \rightarrow+\infty} E_{\lambda}^{0}=1
$$

(ii) Since $e_{\lambda}$ commutes with $T$, we have

$$
\left(\bar{\alpha}\left(1-e_{\lambda}\right) \Psi_{1}, \bar{\alpha} e_{\lambda} \Psi_{2}\right)=0
$$

for all $\Psi_{1}, \Psi_{2} \in \Re$. Hence $\bar{\alpha}\left(1-e_{\lambda}\right) \Psi \perp \mathfrak{Q}_{\lambda}$ and

$$
\begin{aligned}
E_{\lambda}^{0} \bar{\alpha} \Psi & =\bar{\alpha} e_{f(\lambda)} \Psi+E_{\lambda}^{0} \bar{\alpha}\left(1-e_{f(\lambda)}\right) \Psi \\
& =\bar{\alpha} e_{f(\lambda)} \Psi .
\end{aligned}
$$

(iii) For all $\Psi_{1}, \Psi_{2} \in \Re$, we have

$$
\begin{aligned}
d\left(\bar{\alpha} \Psi_{1}, E_{\lambda}^{0} \bar{\alpha} \Psi_{2}\right) & =d\left(\Psi_{1},(1-T) e_{f(\lambda)} \Psi_{2}\right)_{\Re} \\
& =(1+\lambda)^{-1} d\left(\Psi_{1}, e_{f(\lambda)} \Psi_{2}\right)_{\Re} .
\end{aligned}
$$


This also implies that $\bar{\alpha}^{-1} e_{f(\lambda)}$ is bounded for finite $\lambda$ and hence

$$
\bar{\alpha} e_{\lambda} \Re=\mathfrak{S}_{\lambda}, \quad \lambda<1
$$

From (3.7), (3.8), (3.9) and (3.13), we can define a non-negative selfadjoint operator associated with $\mathfrak{R}_{0}$ on $\mathfrak{S}_{0}$ by

$$
B=\int_{0}^{\infty} \lambda^{1 / 2} d E_{\lambda}^{0}
$$

Its domain $D(B)$ is the set of all $\Psi \in \mathfrak{S}$ such that

$$
\left(\|B \Psi\|^{2}=\right) \int_{0}^{\infty} \lambda d\left(\Psi, E_{\lambda}^{0} \Psi\right)<\infty
$$

By (3.15), we have

$$
\begin{aligned}
\left(\|B \bar{\alpha} \Psi\|^{2}\right. & =) \int_{0}^{\infty} \lambda d\left(\bar{\alpha} \Psi, E_{\lambda}^{0} \bar{\alpha} \Psi\right) \\
& =\int_{0}^{\infty} f(\lambda) d\left(\Psi, e_{f(\lambda)} \Psi\right)_{\Omega}=(\Psi, T \Psi)_{\Omega}<\infty
\end{aligned}
$$

and hence $\bar{\alpha} \Re \subset D(B)$. Further, by (3.11), (3.6) and (3.18),

$$
\|\Psi\|_{\stackrel{2}{2}}=\|B \bar{\alpha} \Psi\|^{2}+\|\bar{\alpha} \Psi\|^{2} .
$$

Since the union of (3.16) is dense in $D(B)$ relative to the metric $\left\{\|B \Psi\|^{2}\right.$ $\left.+\|\Psi\|^{2}\right\}^{1 / 2}$ and since $\bar{\alpha} \Re$ is complete relative to the same metric due to (3.19), we have

$$
D(B)=\bar{\alpha} \Omega .
$$

By polarization, we obtain from (3.18),

$$
\left(B \bar{\alpha} \Psi_{1}, B \bar{\alpha} \Psi_{2}\right)=\left(\Psi_{1}, T \Psi_{2}\right)_{\Omega} .
$$

Combining with (3.6), we obtain $\mathfrak{D} \subset D\left(B^{2}\right)$ and

$$
B^{2} \Psi=S \Psi, \Psi \in \mathfrak{D}
$$

Hence 


$$
A_{0}=B^{2}=\int_{0}^{\infty} \lambda d E_{\lambda}^{0}
$$

satisfies $E_{\lambda}^{0} \in \Re_{0}$ and $z=A_{0} x_{0}$.

If $\lim _{\lambda \downarrow 0} E_{\lambda}^{0} \Psi=\Psi$, then

$$
\begin{aligned}
(Q z, \Psi) & =\left(S Q x_{0}, \Psi\right) \\
& =\left(Q x_{0}, A_{0} \Psi\right)=0
\end{aligned}
$$

Since $z$ is assumed to be separating for $\Re_{0}$ and hence is cyclic for $\Re_{0}^{\prime}$, we have $\Psi=0$. Therefore

$$
\lim _{\lambda \downarrow 0} E_{\lambda}^{0}=0
$$

Q.E.D.

Remark. $A_{0}$ satisfying $E_{\lambda}^{0} \in \Re_{0}$ and $z=A_{0} x_{0}$ can be constructed exactly in the same way even if $z$ is not separating for $\Re_{0}$, except that $\lim _{\lambda \downarrow 0} E_{\lambda}^{0}$ is in general a non-zero projection.

In the present case, $A_{0} \geqq 0$ and hence the equality in (3.1) holds only if $Q x_{0}=0$, namely $Q=0$. Therefore $z$ is separating for $\Re_{0}^{\prime}$ and hence is cyclic for $\Re_{0}$.

\section{§4. Proof of Main Theorems}

The unique decompositions $\mu=\mu_{1}+\mu_{2}$ and $\nu=\nu_{1}+\nu_{2}$ are essentially given by the following lemma.

Lemma 4. Let $\Re_{2}$ be a von Neumann algebra on $\mathfrak{S}$ and let $\Psi$ and $\Phi$ be two vectors in $\mathfrak{S}$ such that

$$
(\Psi, Q \Phi) \geqq 0
$$

for all non-negative self-adjoint $Q$ in $\Re_{2}^{\prime}$. Then there exists the largest projection $E$ in $\Re_{2}^{\prime}$ such that

$$
(\Psi, E \Phi)=0
$$


It satisfies

$$
\begin{gathered}
\omega_{\Psi}=\omega_{E \Psi}+\omega_{(1-E) \Psi}, \omega_{\varpi}=\omega_{E \varpi}+\omega_{(1-E) \Phi}, \\
s\left(\omega_{E \Psi}\right) \perp s\left(\omega_{(1-E) \varpi}\right), s\left(\omega_{(1-E) \Psi}\right) \perp s\left(\omega_{E \varpi}\right) \\
s\left(\omega_{E \Psi}\right) \perp s\left(\omega_{E \varpi}\right) .
\end{gathered}
$$

$\omega_{E \emptyset}$ is the largest $\rho \in S\left(\Re_{2}\right)$ such that $\omega_{\emptyset} \geqq \rho$ and $s(\rho) \perp s\left(\omega_{\Psi}\right) . \quad \omega_{E \Psi}$ is the largest $\rho \in S\left(\Re_{2}\right)$ such that $\omega_{\Psi} \geqq \rho$ and $s(\rho) \perp s\left(\omega_{\emptyset}\right)$.

Proof. Let $(\Psi, Q \Phi)=0$ for $Q \in \Re_{2}^{\prime}, Q \geqq 0$. Let $e_{\lambda} \in \Re_{2}^{\prime}$ be the spectral projection of $Q$ (Sakai [6]) and

$$
e(n)=e_{1 /(n-1)}-e_{1 / n}
$$

where $e_{\infty}=1$ and $n=1,2, \ldots$. Since

$$
Q \geqq Q e(n) \geqq n^{-1} e(n),
$$

we have

$$
(\Psi, e(n) \Phi)=0
$$

Hence $(\Psi, Q \Phi)=0$ for $Q \in \Re_{2}^{\prime}$ implies

$$
(\Psi, s(Q) \Phi)=0, s(Q)=\sum_{n} e(n)
$$

For a finite number of projections $E_{i} \in \Re_{2}^{\prime}$, satisfying $\left(\Psi, E_{i} \Phi\right)=0$, we obtain from (4.6)

$$
\left(\Psi, \bigvee_{i} E_{i} \Phi\right)=\left(\Psi, s\left(\sum_{i} E_{i}\right) \Phi\right)=0
$$

From the normality, the same holds for any number of $E_{i}$. Let $E$ be the supremum of $E_{\alpha} \in \Re_{2}^{\prime}$ satisfying $\left(\Psi, E_{\alpha} \Phi\right)=0$. Then, by (4.7), we have $(\Psi, E \Phi)=0$, and by construction, $E$ is the largest such projection in $\mathfrak{R}_{2}^{\prime}$.

From $E \in \Re_{2}^{\prime}$, we have (4.3). From Schwarz inequality for positive linear functional $(\Psi, Q \Phi)$, we have

$$
\left(Q_{1} \Psi, Q_{2} E \Phi\right)=0
$$


for any $Q_{1}, Q_{2} \in \Re_{2}^{\prime}$. Setting $Q_{1}=Q_{3} E$ or $Q_{1}=Q_{3}(1-E)$, we obtain $s\left(\omega_{E \varpi}\right)$ $\perp s\left(\omega_{E \Psi}\right)$ and $s\left(\omega_{E \Phi}\right) \perp s\left(\omega_{(1-E) \Psi}\right)$. Interchanging the role of $\Psi$ and $\Phi$, we obtain $s\left(\omega_{E \Psi}\right) \perp s\left(\omega_{(1-E) \emptyset}\right)$.

Let $\rho \in S\left(\Re_{2}\right)$ be such that

$$
\rho \leqq \omega_{\oplus}, s(\rho) \perp s\left(\omega_{T}\right)
$$

Then there exists $Q \in \mathfrak{R}_{2}^{\prime}, 1 \geqq Q \geqq 0$ satisfying

$$
\rho=\omega_{Q \emptyset}
$$

due to $\rho \leqq \omega_{\varpi}$. Since $\rho\left(s\left(\omega_{\Psi}\right)\right)=0$, we have $s(\Psi) Q \Phi=0$. Hence $(\Psi, Q \Phi)$ $=0$, which implies by $(4.6)$

$$
s(Q) \leqq E
$$

and we have $Q \Phi=E Q \Phi=Q E \Phi$. Therefore

$$
\rho=\omega_{Q \varpi}=\omega_{Q E \emptyset} \leqq \omega_{E \varpi} .
$$

This proves that $\omega_{E \emptyset}$ is the largest $\rho$ satisfying (4.8).

The same proof holds for $\omega_{E T}$.

Q.E.D.

Proof of Theorem 1 (1). By Lemma 1, there exists a representation $\pi_{\mu}$ of $\mathfrak{R}$ on $\mathfrak{S}_{\mu}$ and vectors $\Psi$ and $\Phi \in \mathfrak{S}_{\mu}$ such that

$$
\omega_{T}=\mu, \omega_{\mathscr{\omega}}=\nu, d(\mu, \nu)=\|\Psi-\Phi\|^{2} .
$$

We shall show that for $Q \in \pi(\Re)^{\prime}, Q \geqq 0$

$$
(\Psi, Q \Phi) \geqq 0
$$

This will prove Theorem 1 (1) due to Lemma 4 , where $\Re_{2}=\pi_{\mu}(\Re), \mathfrak{S}=$ $\mathfrak{Q}_{\mu}$.

Suppose $E^{\prime}$ is a projection in $\pi(\Re)^{\prime}$ and $\left(\Psi, E^{\prime} \Phi\right)$ is not a non-negative real number. Then there exists real numbers $\theta_{1}$ and $\theta_{2}$ such that $\theta_{1}$ is not an integer multiple of $2 \pi$ and

$$
\alpha \equiv\left(\Psi, e^{i \theta_{1}} E^{\prime} \Phi\right) \geqq 0, \beta \equiv\left(\Psi, e^{i \theta_{2}}\left(1-E^{\prime}\right) \Phi\right) \geqq 0
$$


Then

$$
\operatorname{Re}(\Psi, \Phi)<\alpha+\beta
$$

Now consider the representation $\pi \oplus \pi$ of $\mathfrak{R}$ on $\mathfrak{g} \oplus \mathfrak{S}$ and vectors

$$
\begin{aligned}
& \Psi^{\prime}=E^{\prime} \Psi \oplus\left(1-E^{\prime}\right) \Psi, \\
& \Phi^{\prime}=e^{i \theta_{1}} E^{\prime} \Phi \oplus e^{i \theta_{2}}\left(1-E^{\prime}\right) \Phi .
\end{aligned}
$$

They satisfy $\omega_{\Psi^{\prime}}=\omega_{\Psi}=\mu, \omega_{\emptyset^{\prime}}=\omega_{\circledast}=\nu$ and, by $(4.10)$,

$$
\left\|\Psi^{\prime}-\Phi^{\prime}\right\|^{2}=\mu(1)+\nu(1)-2(\alpha+\beta)<\|\Psi-\Phi\|^{2},
$$

which is a contradiction with the minimality of $\|\Psi-\Phi\|^{2}$.

Therefore $\left(\Psi, E^{\prime} \Phi\right) \geqq 0$ for any projection $E^{\prime}$ in $\pi(\Re)^{\prime}$ and hence (4.9) holds for any $Q \geqq 0, Q \in \pi(\Re)^{\prime}$.

Q.E.D.

To apply Lemma 3, we need a further reduction:

Lemma 5. Let $\mathfrak{R}_{1}$ be a von Neumann algebra on $\mathfrak{Q}_{1}$ and let $x_{1}$ and $y_{1}$ be vectors in $\mathfrak{Q}_{1}$. Let

$$
P \equiv s\left(s\left(x_{1}\right) s\left(y_{1}\right)\right)
$$

\section{Then}

$$
P \equiv s\left(x_{1}\right) \bigvee s\left(y_{1}\right)-s\left(x_{1}\right) \wedge\left(1-s\left(y_{1}\right)\right)-s\left(y_{1}\right) \wedge\left(1-s\left(x_{1}\right)\right)
$$

Let

$$
\begin{aligned}
& x_{0} \equiv P x_{1}=x_{1}-\left\{s\left(x_{1}\right) \wedge\left(1-s\left(y_{1}\right)\right)\right\} x_{1}, \\
& y_{0} \equiv P y_{1}=y_{1}-\left\{s\left(y_{1}\right) \wedge\left(1-s\left(x_{1}\right)\right)\right\} y_{1} .
\end{aligned}
$$

Then

$$
\begin{gathered}
s\left(x_{0}\right)=s\left(x_{1}\right)-s\left(x_{1}\right) \wedge\left(1-s\left(y_{1}\right)\right), \\
s\left(y_{0}\right)=s\left(y_{1}\right)-s\left(y_{1}\right) \wedge\left(1-s\left(x_{1}\right)\right), \\
s\left(x_{0}\right) \bigvee s\left(y_{0}\right)=P,
\end{gathered}
$$




$$
\begin{gathered}
s\left(x_{0}\right) \wedge\left(1-s\left(y_{0}\right)\right)=0, s\left(y_{0}\right) \wedge\left(1-s\left(x_{0}\right)\right)=0, \\
\left(x_{1}, Q y_{1}\right)=\left(x_{0}, Q y_{0}\right)
\end{gathered}
$$

for all $Q \in \Re_{1}^{\prime}$.

If

$$
\left(x_{1}, Q y_{1}\right)>0
$$

holds for all $Q \in \Re_{1}^{\prime}, Q \geqq 0, Q \neq 0$, then both $x_{0}$ and

$$
z=s\left(x_{0}\right) y_{0}=s\left(x_{1}\right) y_{0}=s\left(x_{1}\right) y_{1}
$$

are cyclic and separating for the restriction

$$
s\left(x_{0}\right) \Re_{1} s\left(x_{0}\right) \equiv \Re_{0}
$$

of $\mathfrak{R}_{1}$ in $s\left(x_{0}\right) \mathfrak{Q}_{1} \equiv \mathfrak{L}_{0}$.

Proof. $s\left(x_{1}\right) s\left(y_{1}\right) \Psi=0$ implies

$$
s\left(y_{1}\right) \Psi \in\left(1-s\left(x_{1}\right)\right) \mathfrak{S}_{1}
$$

and hence

$$
\begin{aligned}
\Psi & =\left(1-s\left(y_{1}\right)\right) \Psi+s\left(y_{1}\right) \Psi \\
& \in\left(1-s\left(y_{1}\right)\right) \mathfrak{S}_{1}+\left\{s\left(y_{1}\right) \mathfrak{L}_{1} \cap\left(1-s\left(x_{1}\right)\right) \mathfrak{L}_{1}\right\} .
\end{aligned}
$$

The converse is also true. Therefore

$$
\operatorname{ker} s\left(x_{1}\right) s\left(y_{1}\right)=\left(1-s\left(y_{1}\right)\right) \mathfrak{S}_{1}+\left\{s\left(y_{1}\right) \wedge\left(1-s\left(x_{1}\right)\right)\right\} \mathfrak{S}_{1} \text {. }
$$

Similar formula holds for $s\left(y_{1}\right) s\left(x_{1}\right)$. Since

$$
(1-P) \mathfrak{S}_{1}=\operatorname{ker} s\left(x_{1}\right) s\left(y_{1}\right) \cap \operatorname{ker}\left(s\left(x_{1}\right) s\left(y_{1}\right)\right)^{*}
$$

by definition, we obtain (4.12). (4.13) and (4.14) then follow.

From (4.13), the set of $Q x_{0}, Q \in \Re_{1}^{\prime}$ is the same as $s\left(x_{1}\right)-s\left(x_{1}\right) \wedge$ $\left(1-s\left(y_{1}\right)\right)$ times the set of $Q x_{1}, Q \in \Re_{1}^{\prime}$ and the set of $Q x_{1}, Q \in \Re_{1}^{\prime}$ spans $s\left(x_{1}\right) \mathfrak{S}_{1}$. Hence we obtain (4.15). Similarly we have (4.16). (4.17) and (4.18) then follow. 
Since $Q y_{1} \in s\left(y_{1}\right) \mathfrak{S}_{1}$ for $Q \in \Re_{1}^{\prime}$, we have

$$
\left(x_{1}, Q y_{1}\right)=\left(x_{0}, Q y_{1}\right) \text {. }
$$

Since $Q x_{0} \in s\left(x_{0}\right) \mathfrak{L}_{1} \subset s\left(x_{1}\right) \mathfrak{L}_{1}$, we have

$$
\left(x_{0}, Q y_{1}\right)=\left(Q^{*} x_{0}, y_{1}\right)=\left(Q^{*} x_{0}, y_{0}\right)
$$

Therefore (4.19) holds.

If (4.20) holds, then for any $Q \in \Re_{1}^{\prime}, Q \geqq 0, Q \neq 0$, we have, by (4.19),

$$
\left(x_{0}, Q y_{0}\right)=\left(x_{0}, Q s\left(x_{0}\right) y_{0}\right)>0
$$

and hence $Q x_{0} \neq 0, Q s\left(x_{0}\right) y_{0} \neq 0$. Therefore $x_{0}$ and $s\left(x_{0}\right) y_{0}$ are separating for $\Re_{1}^{\prime}$. $\quad\left(Q \in \Re_{1}^{\prime}\right.$ and $Q x_{0}=0$ implies $Q^{*} Q x_{0}=0$, hence $Q^{*} Q=0$.) Therefore both $x_{0}$ and $s\left(x_{0}\right) y_{0}$ are cyclic for $\Re_{1}$ and hence cyclic for $s\left(x_{0}\right) \Re_{1} s\left(x_{0}\right)$ on $s\left(x_{0}\right) \mathfrak{S}_{1}$ 。 $x_{0}$ is obviously cyclic for $s\left(x_{0}\right) \Re_{1}^{\prime}$ on $s\left(x_{0}\right) \mathfrak{S}_{1}$ and hence is separating for $s\left(x_{0}\right) \Re_{1} s\left(x_{0}\right)$.

Suppose that $Q \in s\left(x_{0}\right) \Re_{1} s\left(x_{0}\right)$ and

$$
Q s\left(x_{0}\right) y_{0}=0 \text {. }
$$

Then $s\left(Q^{*} Q\right) \leqq 1-s\left(y_{0}\right)$ because $Q y_{0}=Q s\left(x_{0}\right) y_{0}=0$. Since $s\left(Q^{*} Q\right) \leqq$ $s\left(x_{0}\right)$, we have by (4.18)

$$
s\left(Q^{*} Q\right) \leqq s\left(x_{0}\right) \wedge\left(1-s\left(y_{0}\right)\right)=0
$$

Therefore we have $Q=0$. Hence $z=s\left(x_{0}\right) y_{0}$ is separating for $s\left(x_{0}\right) \Re_{1}$ $s\left(x_{0}\right)$.

Q.E.D.

Proof of Theorem 1(2). In the proof of Theorem 1(1), we set

$$
\begin{aligned}
& \mathfrak{S}_{1}=(1-E) \mathfrak{S}_{\mu}, \mathfrak{R}_{1}=\pi_{\mu}(\Re)(1-E), \\
& x_{1}=(1-E) \Psi, \quad y_{1}=(1-E) \Phi,
\end{aligned}
$$

where $E$ is taken from Lemma 4 .

If $Q \in(1-E) \pi_{\mu}(\Re)^{\prime}(1-E), Q \geqq 0, Q \neq 0$, we have 


$$
(\Psi, Q \Phi) \neq 0
$$

due to the maximality of $E$. Therefore we have (4.20).

We now apply Lemma 3 to $\mathfrak{L}_{0}, \Re_{0}, x_{0}$ and $z$ of Lemma 4 , and obtain a positive self-adjoint operator (3.2), where

$$
E_{\lambda}^{0} \in \Re_{0}=s\left(x_{0}\right) \Re_{1} s\left(x_{0}\right) \subset \Re_{1} .
$$

By $\omega_{x_{1}}=\mu_{1}, \pi_{\mu}(Q)(1-E)$ is faithful certainly for

$$
Q \in s\left(\mu_{1}\right) \Re s\left(\mu_{1}\right)
$$

and

$$
\pi_{\mu}\left(s\left(\mu_{1}\right)\right)(1-E)=s\left(x_{1}\right)(1-E)
$$

because $x_{1}$ is cyclic for $\Re_{1}$ on $\mathfrak{S}_{1}$ due to $(4.20)$. Therefore there exists a unique $E_{\lambda}^{1}$ such that for $\lambda>0\left(1-s\left(\mu_{1}\right)\right) \leqq E_{\lambda}^{1}$ and

$$
(1-E) \pi_{\mu}\left(E_{\lambda}^{1}\right)=E_{\lambda}^{0}(1-E)
$$

By the faithfulness of $(1-E) \pi_{\mu}$, we have

(1) $E_{\lambda}^{1} \geqq E_{\lambda^{\prime}}^{1} \quad$ for $\lambda \geqq \lambda^{\prime}$,

(2) $\lim _{\lambda_{n} \uparrow \lambda} E_{\lambda_{n}}^{1}=E_{\lambda}^{1}$

(3) $E_{0}^{1}=0, \lim _{\lambda \uparrow \infty} E_{\lambda}^{1}=1$.

We now define $A_{1}(\nu / \mu)$ by (1.4). We have

$$
\mu_{1}\left(A_{1} Q A_{1}\right)=\left(\pi_{\mu}\left(A_{1}\right) x_{1}, \pi_{\mu}(Q) \pi_{\mu}\left(A_{1}\right) x_{1}\right), Q \in \Re .
$$

Since $\pi_{\mu}\left(E_{\lambda}^{1}\right) x_{1}=E_{\lambda}^{0} x_{1}$ with $E_{\lambda}^{0} \in s\left(x_{0}\right) \Re \Re_{1} s\left(x_{0}\right)$, we have

$$
\pi_{\mu}\left(A_{1}\right) x_{1}=A_{0} x_{0}=s\left(x_{0}\right) y_{1}=s\left(x_{1}\right) y_{1} .
$$

By $\omega_{y_{1}}=\nu_{1}$ and (4.23), we obtain

$$
\left(s\left(x_{1}\right) y_{1}, \pi_{\mu}(Q) s\left(x_{1}\right) y_{1}\right)=\nu_{1}\left(s\left(\mu_{1}\right) Q s\left(\mu_{1}\right)\right) .
$$

By the same argument as for (4.23), we obtain

$$
\pi_{\mu}\left(s\left(\nu_{1}\right)\right)(1-E)=s\left(y_{1}\right)(1-E) .
$$


From (4.15), (4.23) and (4.26), we have

$$
\pi_{\mu}\left(s_{\mu}^{\nu}\right)(1-E)=s\left(x_{0}\right)(1-E) .
$$

Therefore we also have

$$
\left(s\left(x_{0}\right) y_{1}, \pi_{\mu}(Q) s\left(x_{0}\right) y_{1}\right)=\nu_{1}\left(s_{\mu}^{\nu} Q s_{\mu}^{\nu}\right) .
$$

By (4.24), (4.25), and (4.28), we obtain (1.5).

From (4.27), we have

$$
\lim _{\lambda \downarrow 0} E_{\lambda}^{1}=1-s_{\mu}^{\nu}
$$

Proof of Theorem 1 (3). Since the initial assumptions are symmetric in $\mu$ and $\nu$, we define

$$
A_{2}(\mu / \nu) \equiv \int_{0}^{\infty} \lambda^{-1} d E_{\lambda}^{1} s\left(x_{0}\right)
$$

and prove the corresponding properties. By definition of $E_{\lambda}^{1}$,

$$
\pi_{\mu}\left(A_{2}(\mu / \nu) A_{1}(\nu / \mu)\right)^{-}(1-E)=s\left(x_{0}\right)(1-E),
$$

where unbounded operators $A_{k}$ are always defined as the limit of $A_{k} E_{L}^{k}$. By (4.27), we have

$$
\left(A_{2}(\mu / \nu) A_{1}(\nu / \mu)\right)^{-} s_{\mu}^{\nu}
$$

Since $s\left(\nu_{2}\right) \perp s(\mu) \geqq s_{\mu}^{\nu}$, we have $\pi_{\mu}\left(A_{2}(\mu / \nu)\right) E \Phi=0$. Hence, by using $\pi_{\mu}\left(s_{\mu}^{\nu}\right) y_{1}=s\left(x_{0}\right) y_{1}$ and $\pi_{\mu}\left(s_{\mu}^{\nu}\right) x_{1}=x_{0}$,

$$
\begin{aligned}
\pi_{\mu}\left(A_{2}(\mu / \nu)\right) \Phi & =\pi_{\mu}\left(A_{2}(\mu / \nu)\right) y_{1} \\
& =\pi_{\mu}\left(A_{2}(\mu / \nu)\right) s\left(x_{0}\right) y_{1} \\
& =x_{0}=\pi_{\mu}\left(s_{\mu}^{\nu}\right) x_{1} .
\end{aligned}
$$

Therefore we have

$$
\nu\left(A_{2}(\mu / \nu) Q A_{2}(\mu / \nu)\right)=\mu_{1}\left(s_{\mu}^{\nu} Q s_{\mu}^{\nu}\right) .
$$


Proof of Theorem 1 (4). We have already the existence because the vector $y_{1}$ in the proof of Theorem 1 (2) satisfies all requirements. To prove the uniqueness, suppose that $y^{\prime} \in \mathfrak{S}_{1}$ satisfies $\omega_{y^{\prime}}=\nu_{1}$ and $s\left(x_{1}\right) y^{\prime}=$ $z$. Then there exists a partial isometry $u \in \Re_{1}^{\prime}$ such that $u^{*} u y_{1}=y_{1}$ and $u y_{1}=y^{\prime}$ due to $\omega_{y^{\prime}}=\omega_{y_{1}}$. We have

$$
u s\left(x_{1}\right) y_{1}=s\left(x_{1}\right) u y_{1}=s\left(x_{1}\right) y^{\prime}=s\left(x_{1}\right) y_{1}
$$

and hence $u-1$ is 0 on $z$. By applying $\pi_{\mu}\left(A_{2}(\mu / \nu)\right)$, we have

$$
(u-1) x_{0}=0 .
$$

Since $x_{0}$ is separating for $\Re_{1}^{\prime}$, we have $u=1$ and $y^{\prime}=y$. Hence the uniqueness.

Proof of Theorem 2. From the construction of $A_{1}$ and $A_{2}$, we have

$$
\begin{aligned}
d(\mu, \nu)^{2} & =\mu(1)+\nu(1)-2\left(x_{1}, y_{1}\right) \\
& =\mu(1)+\nu(1)-2 \mu_{1}\left(A_{1}\right) \\
& =\mu(1)+\nu(1)-2 \mu\left(A_{2}\right) .
\end{aligned}
$$

To prove the uniqueness, suppose $\Psi_{1}$ and $\Phi_{1}$ be given, satisfying $\omega_{\Psi_{1}}$ $=\mu, \omega_{\sigma_{1}}=\nu$ and $d(\mu, \nu)=\left\|\Psi_{1}-\Phi_{1}\right\|$. By expanding the representation, we can identify $\Psi_{1}$ with $\Psi$ in the proof of Theorem 1 (2), where the representation contains $\pi_{\mu}$ and $\Phi_{1}$ is not necessarily the same as $\Phi$.

Since $\omega_{\emptyset}=\omega_{\Phi_{1}}=\nu$, there exists a partial isometry $u \in \pi(\Re)^{\prime}$, satisfying $u^{*} u \Phi=\Phi$ and $u \Phi=\Phi_{1}$. We also have

$$
(\Psi, \Phi)=d(\mu, \nu)^{1 / 2}=\left(\Psi, \Phi_{1}\right)
$$

Since

$$
\begin{aligned}
s(\Psi) u E \Phi & =u s(\Psi) E \Phi \\
& =u \pi\left(s\left(\omega_{\Psi}\right)\right) E \Phi \\
& =u \pi_{\mu}(s(\mu)) E \Phi=0,
\end{aligned}
$$


we have

$$
\left(\Psi, \Phi_{1}\right)=(\Psi, u(1-E) \Phi)=\left(A_{0}^{1 / 2} x_{1}, u A_{0}^{1 / 2} x_{1}\right)
$$

Equality of this expression with $(\Psi, \Phi)=\left\|A_{0}^{1 / 2} x_{1}\right\|^{2}$ implies

$$
(u-1) A_{0}^{1 / 2} x_{1}=0
$$

By multiplying $\pi\left(A_{2}(\mu / \nu)\right)^{1 / 2}$, we obtain

$$
(u-1) x_{0}=0 .
$$

Since $x_{0}$ is cyclic in $\mathfrak{S}_{1}$, we have $u=1$ on $\mathfrak{S}_{1}$. Therefore

$$
(1-E) \Phi_{1}=y_{1}=(1-E) \Phi \text {. }
$$

Setting $E \Phi_{1}=y^{\prime}$, we obtain the statement of Theorem 2 .

$$
\text { §5. } d_{\pi}(\mu, \nu) \text { and } d(\mu, \nu)
$$

In $[1]$, we have defined

$$
d_{\pi}(\mu, \nu)=\inf \left\{\|\Psi-\Phi\| ; \omega_{\Psi}=\mu, \omega_{\varpi}=\nu, \Psi \in \mathfrak{S}_{\pi}, \Phi \in \mathfrak{S}_{\pi}\right\}
$$

where $\pi$ is a fixed representation on $\mathfrak{S}_{\pi}$. Obviously $d_{\pi}(\mu, \nu) \geqq d(\mu, \nu)$. We shall now discuss when the equality holds.

We shall start by considering whether there exists $\Phi$ giving $d(\mu, \nu)$ $=\|\Psi-\Phi\|$ for the fixed representation $\pi$ and a fixed vector $\Psi$. It already gives some cases where $d(\mu, \nu)=d_{\pi}(\mu, \nu)$.

Theorem 3. Let $\mu, \nu \in S(\Re)$ and $\pi$ be a fixed representation of $\Re$ on $\mathfrak{S}_{\pi}$. Let $\Psi$ be a fixed vector in $\mathfrak{S}_{\pi}$ satisfying $\omega_{\Psi}=\mu$.

(1) Let $E_{1}$ be the projection on the closure of

$$
\pi(\Re) \pi\left(A_{2}(\nu / \mu)\right) \Psi .
$$

Then there exists $\Phi \in \mathfrak{D}_{\pi}$ satisfying

$$
\omega_{\emptyset}=\nu,\|\Psi-\Phi\|=d(\mu, \nu)
$$


if and only if there exists a vector $y^{\prime}$ in $\left(1-E_{1}\right) H$ such that $\omega_{y^{\prime}}=\nu_{2}$.

(2) If $s(\mu) \geqq s(\nu)$, then there always exists $\Phi \in \mathfrak{S}_{\pi}$ satisfying (5.3).

(3) If $\Psi$ is separating for $\pi(\Re)$, there always exists $\Phi \in \mathfrak{S}_{\pi}$ satisfying (5.3).

Proof. We first extend $\pi$ to sufficiently large representation $\hat{\pi}$ of $\Re$ on $\hat{\mathfrak{S}} \in \mathfrak{S}_{\pi}$ such that $\Phi$ in Theorem 1 is in $\hat{\mathfrak{S}}$.

By (4.30) with $\mu$ and $\nu$ exchanged, we have

$$
\pi\left(A_{2}(\mu / \nu)\right) \Psi=\hat{\pi}\left(A_{2}(\mu / \nu)\right) \Psi=y_{0} \in \mathfrak{Q}_{\pi} .
$$

Since $y_{0}$ is cyclic for $\hat{\pi}(\Re)$ on $\mathfrak{S}_{1}$, we have

$$
E_{1} \hat{\mathfrak{S}}=\mathfrak{S}_{1} \subset \mathfrak{S}_{\pi}
$$

Therefore we have (1).

If $s(\mu) \geqq s(\nu)$, then $\nu_{2}=0$ and (2) follows from (1).

If $\Psi$ is separating for $\pi(\Re)$, then $s(\mu)=1$ and $s(\mu) \geqq s(\nu)$ for any $\nu$. Hence (3) follows from (2).

Q.E.D.

Theorem 4. Let $\pi$ be a fixed representation of $\mathfrak{R}$ on $\mathfrak{S}_{\pi}$ and $x, y \in$ $\mathfrak{L}_{\pi}$. Then

$$
d_{\pi}\left(\omega_{x}, \omega_{y}\right)=d\left(\omega_{x}, \omega_{y}\right)
$$

and there exist $\Psi$ and $\Phi$ in $\mathfrak{Q}_{\pi}$ such that

$$
\omega_{\Psi}=\omega_{x}, \omega_{\varpi}=\omega_{y}, d\left(\omega_{x}, \omega_{y}\right)=\|\Psi-\Phi\| .
$$

Proof. Let $\mu=\omega_{x}, \nu=\omega_{y}$ and $E_{1}$ be the projection on the closure of

$$
\pi(\Re) \pi\left(A_{2}(\mu / \nu)\right) x
$$

and $E_{1}^{\prime}$ be the projection on the closure of

$$
\pi(\Re) \pi\left(A_{2}(\nu / \mu)\right) y .
$$

Since $\pi(\Re)$ on $E_{1} \mathfrak{S}_{\pi}$ and $E_{1}^{\prime} \mathfrak{Q}_{\pi}$ are unitarily equivalent by the uniqueness in Theorem 1 (4), there exists a partial isometry $u \in \pi(\Re)^{\prime}$ such that 


$$
\begin{gathered}
u^{*} u=E_{1}, u u^{*}=E_{1}^{\prime}, \\
\left(x, u^{*} y\right)=\omega_{x}\left(A_{2}(\nu / \mu)\right)
\end{gathered}
$$

There exist a central projection $F$ and partial isometries $u_{1}, u_{2} \in \pi(\Re)^{\prime}$ such that

$$
\begin{gathered}
u_{1}^{*} u_{1}=F\left(1-E_{1}\right), \quad u_{1} u_{1}^{*} \leqq F\left(1-E_{1}^{\prime}\right), \\
u_{2}^{*} u_{2} \leqq(1-F)\left(1-E_{1}\right), u_{2} u_{2}^{*}=(1-F)\left(1-E_{1}^{\prime}\right) .
\end{gathered}
$$

We set

$$
\begin{gathered}
\Psi=F\left(u_{1}+u\right) x+(1-F) x \\
\Phi=F y+(1-F)\left(u_{2}^{*}+u^{*}\right) y .
\end{gathered}
$$

Since $F$ is a central projection, we have

$$
\omega_{\Psi}=\omega_{x_{F}}+\omega_{(1-F) x}, x_{F} \equiv F\left(u_{1}+u\right) x
$$

Since $E_{1}^{\prime} u=u,\left(1-E_{1}^{\prime}\right) u_{1}=u_{1}$, we have $u^{*} u_{1}=u_{1}^{*} u=0$. Therefore

$$
F\left(u_{1}+u\right)^{*}\left(u_{1}+u\right)=F\left(u_{1}^{*} u_{1}+u^{*} u\right)=F .
$$

Since $u_{1}+u \in \pi(\Re)^{\prime}$, we have

$$
\omega_{x_{F}}=\omega_{F x}, \omega_{F}=\omega_{F x}+\omega_{(1-F) x}=\omega_{x} .
$$

Similarly, we have

$$
\omega_{\emptyset}=\omega_{y}
$$

Since

$$
\omega_{u_{1} x}=\omega_{F\left(1-E_{1}\right) x} \leqq \omega_{\left(1-E_{1}\right) x}=\omega_{x}-\omega_{E_{1} x}=\mu_{2}(x),
$$

we obtain

$$
s\left(u_{1} x\right) \leqq \pi\left(s\left(\mu_{2}\right)\right) \perp s(\nu) .
$$

Hence 


$$
\begin{aligned}
\left(F u_{1} x, F y\right) & =\left(F \pi\left(s\left(\mu_{2}\right)\right) u_{1} x, F y\right)=\left(F u_{1} x, F \pi\left(s\left(\mu_{2}\right)\right) y\right) \\
= & 0
\end{aligned}
$$

Similarly

$$
\left((1-F) x,(1-F) u_{2}^{*} y\right)=0 .
$$

Therefore

$$
\begin{aligned}
(\Psi, \Phi) & =(F u x, F y)+\left((1-F) x,(1-F) u^{*} y\right)=\left(x, u^{*} y\right) \\
& =\omega_{x}\left(A_{2}(\nu / \mu)\right) .
\end{aligned}
$$

Remark. By $[2], d\left(\omega, \omega^{\prime}\right)^{2} \leqq\left\|\omega-\omega^{\prime}\right\|$. Using (5.4), we then have

$$
d_{\pi}\left(\omega_{x}, \omega_{y}\right)^{2} \leqq\left\|\omega_{x}-\omega_{y}\right\|
$$

The remark at the end of $[1]$ is thus incorrect. The counterexample mentioned there was a counter-example only to the method of $[1]$.

\section{§6. Discussions}

If $\mu \geqq \nu$, then we can obtain $\left\|A_{2}\right\| \leqq 1$ as follows. From $\mu \geqq \nu$, we have

$$
0=\mu(1-s(\mu)) \geqq \nu(1-s(\mu))
$$

and hence $s(\mu) \geqq s(\nu)$. Thus $\nu_{2}=0, s_{\nu}^{\mu}=s(\nu)$ and

$$
\nu_{1}(Q)=\nu(Q)=\mu\left(A_{2} Q A_{2}\right) .
$$

Let $b>1$. Then

$$
\begin{aligned}
\nu\left(1-E_{b}^{2}\right) & =\mu\left(\left(1-E_{b}^{2}\right) A_{2}^{2}\right) \geqq b^{2} \mu\left(1-E_{b}^{2}\right) . \\
& \geqq b^{2} \nu\left(1-E_{b}^{2}\right) .
\end{aligned}
$$

Therefore $\nu\left(1-E_{b}^{2}\right)=0$ and hence $\mu\left(1-E_{b}^{2}\right)=0$. This implies $E_{b}^{2} \geqq s(\mu)$. Since $s_{\nu}^{\mu} A_{2}=A_{2}$ and $s(\mu) \geqq s_{\nu}^{\mu}$, we have $\left(1-E_{b}^{2}\right) A_{2}=0$ and hence $\left\|A_{2}\right\|=1$. From (6.1), we see that $A_{2}$ is the same as Sakai's $t_{0}$. 
As for $A_{1}$, we have for $Q_{1}, Q_{2} \in \pi_{1}(\Re)^{\prime}$

$$
\begin{aligned}
\left(Q_{1} x_{0}, \pi_{1}\left(A_{2}\right) Q_{2} x_{0}\right) & =\left(Q_{1} x_{0}, Q_{2} \pi_{1}\left(A_{2}\right) \pi_{1}\left(s_{\nu}^{\mu}\right) x_{0}\right) \\
& =\left(Q_{1} x_{0}, Q_{2} y_{0}\right)=\left(Q_{1} x_{0}, Q_{2} s\left(x_{0}\right) y_{0}\right) \\
& =\left(Q_{1} x_{0}, Q_{2} z\right)=\left(Q_{1} x_{0}, \pi_{1}\left(A_{1}\right) Q_{2} x_{0}\right) .
\end{aligned}
$$

Therefore we have, from $s\left(x_{0}\right) \pi_{1}\left(A_{1}\right)=\pi_{1}\left(A_{1}\right)$,

$$
\pi_{1}\left(A_{1}\right)=s\left(x_{0}\right) \pi_{1}\left(A_{2}\right) s\left(x_{0}\right)=\pi_{1}\left(s_{\mu}^{\nu} A_{2} s_{\mu}^{\nu}\right)
$$

and hence

$$
A_{1}=s_{\mu}^{\nu} A_{2} s_{\mu}^{\nu}
$$

In particular $\left\|A_{1}\right\| \leqq 1$.

If $\Re$ is commutative, $\mu, \nu \in S(\Re)$ are measures on the spectrum of $\Re$ and $A_{1}=A_{2}$ is the square root of the Radon-Nikodym derivative. The decomposition $\nu=\nu_{1}+\nu_{2}$ is the decomposition of the measure $\nu$ into absolutely continuous and singular parts relative to the measure $\mu$.

From proof of Theorem 1 , it is seen that $\pi_{\mu}\left(A_{1}\right)$ is unique on (1$\left.s\left(x_{0}\right)\right) \mathfrak{S}_{1}+\mathfrak{D}$. We would obtain the uniqueness of $A_{1}$ if $\mathfrak{D}$ is the core of $A_{0}$ in Lemma 3.

As for the appearance of two operators $A_{1}$ and $A_{2}$, we have the following result.

Theorem 5. $A_{1}$ and $A_{2}$ coincide if and only if $s\left(\mu_{1}\right)$ commutes with $s\left(\nu_{1}\right)$. If $s\left(\mu_{1}\right)$ and $s\left(\nu_{1}\right)$ commute, then $s_{\mu}^{\mu}=s_{\nu}^{\mu}=s\left(\mu_{1}\right) s\left(\nu_{1}\right)$.

Proof. From the construction of $A_{1}$ and $A_{2}$ in the proof of Theorem 1 , it is clear that $A_{1}=A_{2}$ if and only if $s_{\mu}^{\nu}=s_{\nu}^{\mu}$.

If $s\left(\mu_{1}\right)$ and $s\left(\nu_{1}\right)$ commute, then obviously $s_{\mu}^{\nu}=s_{\nu}^{\mu}=s\left(\mu_{1}\right) s\left(\nu_{1}\right)$. If $s\left(\mu_{1}\right)$ does not commute with $s\left(\nu_{1}\right)$ then $\left[s_{\mu}^{\nu}, s\left(\mu_{1}\right)\right]=0$ while $\left[s\left(\mu_{1}\right), s_{\nu}^{\mu}\right]$ $=\left[s\left(\mu_{1}\right), s\left(\nu_{1}\right)\right] \neq 0$ and hence $s_{\mu}^{\nu} \neq s_{\nu}^{\mu} . \quad\left[\right.$ Note that $s_{\nu}^{\mu}=s\left(\nu_{1}\right)-s\left(\nu_{1}\right) \wedge(1-$ $\left.s(\mu))=s\left(\nu_{1}\right)-s\left(\nu_{1}\right) \wedge\left(1-s\left(\mu_{1}\right)\right).\right] \quad$ Q.E.D.

If $\mu \geqq \nu$, then $[s(\mu), s(\nu)]=0$. The following example gives the 
case where $\mu \geqq \nu$ and $\left[s\left(\mu_{1}\right), s\left(\nu_{1}\right)\right] \neq 0$.

Example. Let $\mathfrak{R}=\mathscr{B}(\mathfrak{S})$ and $p$ and $q$ be mutually orthogonal unit vectors in $\mathfrak{S}$. Let

$$
\mu=\omega_{p}+\omega_{p+q}, \nu=\frac{1}{2} \omega_{q} .
$$

First we prove $\mu \geqq \nu$. For $Q \geqq 0, Q \in \Re$,

$$
\begin{aligned}
\mu(Q) & =(p, Q p)+(p+q, Q(p+q)) \\
& =2(p, Q p)+(q, Q q)+2 \operatorname{Re}(p, Q q) .
\end{aligned}
$$

Since

$$
\begin{aligned}
2|(p, Q q)| & \leqq 2(p, Q p)^{\frac{1}{2}}(q, Q q)^{\frac{1}{2}} \\
& \leqq 2(p, Q p)+\frac{1}{2}(q, Q q)
\end{aligned}
$$

we obtain

$$
\mu(Q) \geqq \frac{1}{2}(q, Q q)=\nu(Q)
$$

Next, we see that any $\rho$ satisfying $\mu \geqq \rho$ and $s(\rho) \perp s(\nu)$ must be proportional to $\omega_{p}$ because $\mu \geqq \rho$ implies $s(\rho) \leqq s(p)+s(q)$ and $(1-s(\nu))$ $\wedge(s(p)+s(q))=s(p)$ due to $s(\nu)=s(q)$. Since $\mu \geqq \omega_{p}$ and $\mu(Q)=\omega_{p}(Q)$ $\neq 0$ if $Q=s(p-q)$, we see that $\mu_{2}=\omega_{p}$ and hence $\mu_{1}=\omega_{p+q}$. Therefore $s\left(\mu_{1}\right)=s(p+q)$ does not commute with $s\left(\nu_{1}\right)=s(\nu)=s(q)$.

In this example $s_{\nu}^{\mu}=s(q), s_{\mu}^{\nu}=s(p+q)$ and $A_{1}=2^{-3 / 2} s(p+q), A_{2}=$ $2^{-1 / 2} s(q)$.

\section{Acknowledgement}

This work is completed during the author's stay at the Dept. of Mathematics, Queen's University. The author would like to thank Professor Coleman and Professor Woods for their warm hospitality. 


\section{References}

[1] Araki, H., Publ. RIMS Kyoto Univ. 6 (1970/71), 477-487.

[2] Bures, D.J.C., Trans. Amer. Math. Soc. 135 (1969), 199-212

[3] Dixmier, J., Les Algèbres d'Opérateurs dans l'Espace Hilbertien, 2nd ed., Gauthier Villars, Paris, 1969.

[4] Dye, H.A., Trans. Amer. Math. Soc. 70 (1952), 243-280.

[5] Sakai, S., Bull. Amer. Math. Soc. 71 (1965), 149-151.

[6] Sakai, S., $C^{*}$-Algebras and $W^{*}$-Algebras, Springer-Verlag, New York, Heidelberg, Berlin, 1971.

[7] Segal, I.E., Ann. of Math. 57 (1953), 401-457.

[8] Takesaki, M., Tomita's Theory of Modular Hilbert Algebras and Its Applications, Springer-Verlag, Berlin, Heidelberg, New York, 1970.

Note Added in Proof. Professor J. Dixmier has pointed out that $1-E$ in Lemma 4 is the support of the normal state $(\Psi, Q \Phi)$ and hence its existence is well-known. 